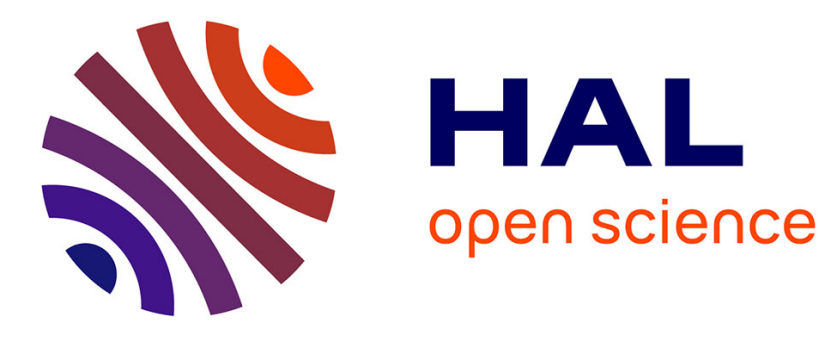

\title{
Median topographic maps for biomedical data sets
}

\author{
Barbara Hammer, Alexander Hasenfuss, Fabrice Rossi
}

\section{To cite this version:}

Barbara Hammer, Alexander Hasenfuss, Fabrice Rossi. Median topographic maps for biomedical data sets. Villmann, Th.; Biehl, M.; Hammer, B.; Verleysen, M. Similarity-Based Clustering, Springer Berlin / Heidelberg, pp.92-117, 2009, Lecture Notes in Computer Science, 10.1007/978-3-642-018053_6. hal-00413148

\section{HAL Id: hal-00413148 https://hal.science/hal-00413148}

Submitted on 3 Sep 2009

HAL is a multi-disciplinary open access archive for the deposit and dissemination of scientific research documents, whether they are published or not. The documents may come from teaching and research institutions in France or abroad, or from public or private research centers.
L'archive ouverte pluridisciplinaire HAL, est destinée au dépôt et à la diffusion de documents scientifiques de niveau recherche, publiés ou non, émanant des établissements d'enseignement et de recherche français ou étrangers, des laboratoires publics ou privés. 


\title{
Median topographic maps for biomedical data sets
}

\author{
Barbara Hammer ${ }^{1}$, Alexander Hasenfuss ${ }^{1}$, and Fabrice Rossi ${ }^{2}$ \\ 1 Clausthal University of Technology, D-38678 Clausthal-Zellerfeld, Germany, \\ ${ }^{2}$ INRIA Rocquencourt, Domaine de Voluceau, Rocquencourt, B.P. 105, 78153 Le \\ Chesnay Cedex, France
}

\begin{abstract}
Median clustering extends popular neural data analysis methods such as the self-organizing map or neural gas to general data structures given by a dissimilarity matrix only. This offers flexible and robust global data inspection methods which are particularly suited for a variety of data as occurs in biomedical domains. In this chapter, we give an overview about median clustering and its properties and extensions, with a particular focus on efficient implementations adapted to large scale data analysis.
\end{abstract}

\section{Introduction}

The tremendous growth of electronic information in biological and medical domains has turned automatic data analysis and data inspection tools towards a key technology for many application scenarios. Clustering and data visualization constitute one fundamental problem to arrange data in a way understandable by humans. In biomedical domains, prototype based methods are particularly well suited since they represent data in terms of typical values which can be directly inspected by humans and visualized in the plane if an additional low-dimensional neighborhood or embedding is present. Popular methodologies include K-means clustering, the self-organizing map, neural gas, affinity propagation, etc. which have successfully been applied to various problems in the biomedical domain such as gene expression analysis, inspection of mass spectrometric data, healthcare, analysis of microarray data, protein sequences, medical image analysis, etc. [1, 37, 36, 41, $44,53,54$.

Many popular prototype-based clustering algorithms, however, have been derived for Euclidean data embedded in a real-vector space. In biomedical applications, data are diverse including temporal signals such as EEG and EKG signals, functional data such as mass spectra, sequential data such as DNA sequences, complex graph structures such as biological networks, etc. Often, the Euclidean metric is not appropriate to compare such data, rather, a problem dependent similarity or dissimilarity measure should be used such as alignment, correlation, graph distances, functional metrics, or general kernels.

Various extensions of prototype-based methods towards more general data structures exist such as extensions for recurrent and recursive data structures, 
functional versions, or kernelized formulations, see e.g. 27,26, 25, 2, 24, for an overview. A very general approach relies on a matrix which characterizes the pairwise similarities or dissimilarities of data. This way, any distance measure or kernel (or generalization thereof which might violate symmetry, triangle inequality, or positive definiteness) can be dealt with including discrete settings which cannot be embedded in Euclidean space such as alignment of sequences or empirical measurements of pairwise similarities without explicit underlying metric.

Several approaches extend popular clustering algorithms such as K-means or the self-organizing map towards this setting by means of the relational dual formulation or kernelization of the approaches $330,31,51,8,24$, . These methods have the drawback that they partially require specific properties of the dissimilarity matrix (such as positive definiteness), and they represent data in terms of prototypes which are given by (possibly implicit) mixtures of training points, thus they cannot easily be interpreted directly. Another general approach leverages mean field annealing techniques 19,20,33 as a way to optimize a modified criterion that does not rely anymore on the use of prototypes. As for the relational and kernel approaches, the main drawback of those solutions is the reduced interpretability.

An alternative is offered by a representation of classes by the median or centroid, i.e. prototype locations are restricted to the discrete set given by the training data. This way, the distance of data points from prototypes is welldefined. The resulting learning problem is connected to a well-studied optimization problem, the K-median problem: given a set of data points and pairwise dissimilarities, find $k$ points forming centroids and an assignment of the data into $\mathrm{k}$ classes such that the average dissimilarities of points to their respective closest centroid is minimized. This problem is NP hard in general unless the dissimilarities have a special form (e.g. tree metrics), and there exist constant factor approximations for specific settings (e.g. metrics) [10,6].

The popular K-medoid clustering extends the batch optimization scheme of K-means to this restricted setting of prototypes: it in turn assigns data points to the respective closest prototypes and determines optimum prototypes for these assignments [38.96]. Unlike K-means, there does not exist a closed form of the optimum prototypes given fixed assignments such that exhaustive search is used. This results in a complexity $\mathcal{O}\left(N^{2}\right)$ for one epoch for K-centers clustering instead of $\mathcal{O}(N)$ for K-means, $N$ being the number of data points. Like K-means, Kcenters clustering is highly sensitive to initialization.

Various approaches optimize the cost function of K-means or K-median by different methods to avoid local optima as much as possible, such as Lagrange relaxations of the corresponding integer linear program, vertex substitution heuristics, or affinity propagation [28, 17.18]. In the past years, simple, but powerful extensions of neural based clustering to general dissimilarities have been proposed which can be seen as generalizations of K-centers clustering to include neighborhood cooperation, such that the topology of data is taken into account. More precisely, the median clustering has been integrated into the popular self- 
organizing map (SOM) [4t and its applicability has been demonstrated in a large scale experiment from bioinformatics 41. Later, the same idea has been integrated into neural gas (NG) clustering together with a proof of the convergence of median SOM and median NG clustering [13]. Like K-centers clustering, the methods require an exhaustive search to obtain optimum prototypes given fixed assignments such that the complexity of a standard implementation for one epoch is $\mathcal{O}\left(N^{2} K\right)$ (this can be reduced to $\mathcal{O}\left(N^{2}+N K^{2}\right)$ for median SOM, see Section 4 and [12]). Unlike K-means, local optima and overfitting can widely be avoided due to the neighborhood cooperation such that fast and reliable methods result which are robust with respect to noise in the data. Apart from this numerical stability, the methods have further benefits: they are given by simple formulas and they are very easy to implement, they rely on underlying cost functions which can be extended towards the setting of partial supervision, and in many situations a considerable speed-up of the algorithm can be obtained, as demonstrated in $[12,29$, for example.

In this chapter, we present an overview about neural based median clustering. We present the principle methods based on the cost functions of NG and SOM, respectively, and discuss applications, extensions and properties. Afterwards, we discuss several possibilities to speed-up the clustering algorithms, including exact methods, as well as single pass approximations for large data sets.

\section{Prototype based clustering}

Prototype based clustering aims for representing given data from some set $X$ faithfully by means of prototypical representatives $\left\{\boldsymbol{w}^{1}, \ldots, \boldsymbol{w}^{K}\right\}$. In the standard Euclidean case, real vectors are dealt with, i.e. $X \subseteq \mathbb{R}^{M}$ and $\boldsymbol{w}^{i} \in \mathbb{R}^{M}$ holds for all $i$ and some dimensionality $M$. For every data point $\boldsymbol{x} \in X$, the index of the winner is defined as the prototype

$$
I(\boldsymbol{x})=\operatorname{argmin}_{j}\left\{d\left(\boldsymbol{x}, \boldsymbol{w}^{j}\right)\right\}
$$

where

$$
d\left(\boldsymbol{x}, \boldsymbol{w}^{j}\right)=\sum_{i=1}^{M}\left(x_{i}-w_{i}^{j}\right)^{2}
$$

denotes the squared Euclidean distance. The receptive field of prototype $\boldsymbol{w}^{j}$ is defined as the set of data points for which it becomes winner. Typically, clustering results are evaluated by means of the quantization error which measures the distortion being introduced when data is represented by a prototype, i.e.

$$
E:=\frac{1}{2} \cdot \int \sum_{j=1}^{K} \delta_{I(\boldsymbol{x}), j} \cdot d\left(\boldsymbol{x}, \boldsymbol{w}^{j}\right) P(\boldsymbol{x}) d \boldsymbol{x}
$$

for a given probability measure $P$ according to which data are distributed. $\delta_{i j}$ denotes the Kronecker function. In many training settings, a finite number of 
data $X=\left\{\boldsymbol{x}^{1}, \ldots, \boldsymbol{x}^{N}\right\}$ is given in advance and the corresponding discrete error becomes

$$
\hat{E}:=\frac{1}{2 N} \cdot \sum_{i=1}^{N} \sum_{j=1}^{K} \delta_{I\left(\boldsymbol{x}^{i}\right), j} \cdot d\left(\boldsymbol{x}^{i}, \boldsymbol{w}^{j}\right)
$$

The popular K-means clustering algorithm aims at a direct optimization of the quantization error. In batch mode, it, in turn, determines optimum prototypes $\boldsymbol{w}^{j}$ given fixed assignments $I\left(\boldsymbol{x}^{i}\right)$ and vice versa until convergence:

$$
k_{i j}:=\delta_{I\left(\boldsymbol{x}^{i}\right), j}, \quad \boldsymbol{w}^{j}:=\frac{\sum_{i} k_{i j} \boldsymbol{x}^{i}}{\sum_{i} k_{i j}}
$$

This update scheme is very sensitive to the initialization of prototypes such that multiple restarts are usually necessary.

\section{Neural gas}

The self-organizing map and neural gas enrich the update scheme by neighborhood cooperation of the prototypes. This accounts for a topological ordering of the prototypes such that initialization sensitivity is (almost) avoided. The cost function of neural gas as introduced by Martinetz [46] has the form

$$
E_{\mathrm{NG}} \sim \frac{1}{2} \cdot \int \sum_{j=1}^{K} h_{\sigma}\left(\operatorname{rk}\left(\boldsymbol{x}, \boldsymbol{w}^{j}\right)\right) \cdot d\left(\boldsymbol{x}, \boldsymbol{w}^{j}\right) P(\boldsymbol{x}) d \boldsymbol{x}
$$

where

$$
\operatorname{rk}\left(\boldsymbol{x}, \boldsymbol{w}^{j}\right)=\left|\left\{\boldsymbol{w}^{k} \mid d\left(\boldsymbol{x}, \boldsymbol{w}^{k}\right)<d\left(\boldsymbol{x}, \boldsymbol{w}^{j}\right)\right\}\right|
$$

denotes the rank of prototype $\boldsymbol{w}^{j}$ sorted according to its distance from the data point $\boldsymbol{x}$ and $h_{\sigma}(t)=\exp (-t / \sigma)$ is a Gaussian shaped curve with the neighborhood range $\sigma>0 . \sigma$ is usually annealed during training. Obviously, $\sigma \rightarrow 0$ yields the standard quantization error. For large values $\sigma$, the cost function is smoothed such that local optima are avoided at the beginning of training. For a discrete training set, this cost term becomes

$$
\hat{E}_{\mathrm{NG}} \sim \frac{1}{2 N} \cdot \sum_{i=1}^{N} \sum_{j=1}^{K} h_{\sigma}\left(\operatorname{rk}\left(\boldsymbol{x}^{i}, \boldsymbol{w}^{j}\right)\right) \cdot d\left(\boldsymbol{x}^{i}, \boldsymbol{w}^{j}\right)
$$

This cost function is often optimized by means of a stochastic gradient descent. An alternative is offered by batch clustering which, in analogy to K-means, consecutively optimizes assignments and prototype locations until convergence, as described in 13:

$$
k_{i j}:=\operatorname{rk}\left(\boldsymbol{x}^{i}, \boldsymbol{w}^{j}\right), \quad \boldsymbol{w}^{j}:=\frac{\sum_{i} h_{\sigma}\left(k_{i j}\right) \boldsymbol{x}^{i}}{\sum_{i} h_{\sigma}\left(k_{i j}\right)}
$$

Neighborhood cooperation takes place depending on the given data at hand by means of the ranks. This accounts for a very robust clustering scheme which is 
very insensitive to local optima, as discussed in 446. Further, as shown in 447 neighborhood cooperation induces a topology on the prototypes which perfectly fits the topology of the underlying data manifold provided the sampling is sufficiently dense. Thus, browsing within this information space becomes possible.

\section{Self-organizing map}

Unlike NG, SOM uses a priorly fixed lattice structure, often a regular lowdimensional lattice such that visualization of data can directly be achieved. Original SOM as proposed by Kohonen [39] does not possess a cost function in the continuous case, but a simple variation as proposed by Heskes does [32]. The corresponding cost function is given by

$$
E_{\mathrm{SOM}} \sim \frac{1}{2} \cdot \int \sum_{j=1}^{K} \delta_{I^{*}(\boldsymbol{x}), j} \cdot \sum_{k=1}^{N} h_{\sigma}(\operatorname{nd}(j, l)) \cdot d\left(\boldsymbol{x}, \boldsymbol{w}^{l}\right) P(\boldsymbol{x}) \boldsymbol{x}
$$

where $\operatorname{nd}(j, l)$ describes the distance of neurons arranged on a priorly chosen neighborhood structure of the prototypes, often a regular two-dimensional lattice, and

$$
I^{*}(\boldsymbol{x})=\operatorname{argmin}_{i}\left\{\sum_{l=1}^{K} h_{\sigma}(\operatorname{nd}(i, l)) d\left(\boldsymbol{x}, \boldsymbol{w}^{l}\right)\right\}
$$

describes the prototype which is closest to $\boldsymbol{x}$ if averaged over the neighborhood. (This is in practice often identical to the standard winner.) In the discrete case, the cost function becomes

$$
\hat{E}_{\mathrm{SOM}} \sim \frac{1}{2 N} \cdot \sum_{i=1}^{N} \sum_{j=1}^{K} \delta_{I^{*}\left(\boldsymbol{x}^{i}\right), j} \cdot \sum_{k=1}^{N} h_{\sigma}(\operatorname{nd}(j, k)) \cdot d\left(\boldsymbol{x}^{i}, \boldsymbol{w}^{k}\right)
$$

SOM is often optimized by means of a stochastic gradient descent or, alternatively, in a fast batch mode, subsequently optimizing assignments and prototypes as follows:

$$
k_{i j}:=\delta_{I^{*}\left(\boldsymbol{x}^{i}\right), j}, \quad \boldsymbol{w}^{j}:=\frac{\sum_{i, k} k_{i k} h_{\sigma}(\operatorname{nd}(k, j)) \boldsymbol{x}^{i}}{\sum_{i, k} k_{i k} h_{\sigma}(\operatorname{nd}(k, j))}
$$

As before, the neighborhood range $\sigma$ is annealed to 0 during training, and the standard quantization error is recovered. Intermediate steps offer a smoothed cost function the optimization of which is simpler such that local optima can widely be avoided and excellent generalization can be observed. Problems can occur if the priorly chosen topology does not fit the underlying manifold and topological mismatches can be observed. Further, topological defomations can easily occur in batch optimization when annealing the neighborhood quickly as demonstrated in [16]. These problems are not present for the data optimum topology provided by NG, but, unlike SOM, NG does not offer a direct visualization of data. Batch SOM, NG, and K-means converge after a finite and usually small number of epochs 13 . 


\section{Median clustering}

Assume data is characterized only by a matrix of pairwise nonnegative dissimilarities

$$
D=\left(d\left(\boldsymbol{x}^{i}, \boldsymbol{x}^{j}\right)\right)_{i, j=1, \ldots, N},
$$

i.e. it is not necessarily contained in an Euclidean space. This setting covers several important situations in biomedical domains such as sequence data which are compared by alignment, time dependent signals for which correlation analysis gives a good dissimilarity measure, or medical images for which problem specific dissimilarity measures give good results. In particular, the data space $X$ is discrete such that prototypes cannot be adapted smoothly within the data space.

The idea of median clustering is to restrict prototype locations to data points. The objective of clustering as stated by the quantization error (4) is well defined if prototypes are restricted to the data set $\boldsymbol{w}^{j} \in X$ and the squared Euclidan metric is substituted by a general term $d\left(\boldsymbol{x}^{i}, \boldsymbol{w}^{j}\right)$ given by a dissimilarity matrix only. Similarly, the cost functions of neural gas and the self-organizing map remain well-defined for $\boldsymbol{w}^{j} \in X$ and arbitrary terms $d\left(\boldsymbol{x}^{i}, \boldsymbol{w}^{j}\right)$. Thereby, the dissimilarities $D$ need not fulfill the conditions of symmetry or the triangle inequality.

One can derive learning rules based on the cost functions in the same way as batch clustering by means of a subsequent optimization of prototype locations and assignments. For NG, optimization of the cost function

$$
\hat{E}_{\mathrm{NG}} \sim \frac{1}{2 N} \cdot \sum_{i=1}^{N} \sum_{j=1}^{K} h_{\sigma}\left(\operatorname{rk}\left(\boldsymbol{x}^{i}, \boldsymbol{w}^{j}\right)\right) \cdot d\left(\boldsymbol{x}^{i}, \boldsymbol{w}^{j}\right)
$$

with the constraint $\boldsymbol{w}^{j} \in X$ yields the following algorithm for median NG:

$$
\begin{aligned}
& \text { init } \\
& \text { repeat } \\
& \qquad \begin{aligned}
k_{i j} & :=\operatorname{rk}\left(\boldsymbol{x}^{i}, \boldsymbol{w}^{j}\right) \\
\boldsymbol{w}^{j} & :=\operatorname{argmin}_{\boldsymbol{x}^{l}} \sum_{i=1}^{N} h_{\sigma}\left(k_{i j}\right) d\left(\boldsymbol{x}^{i}, \boldsymbol{x}^{l}\right)
\end{aligned}
\end{aligned}
$$

Unlike batch NG, a closed solution for optimum prototype locations does not exist and exhaustive search is necessary. In consequence, one epoch has time complexity $\mathcal{O}\left(N^{2} K\right)$ compared to $\mathcal{O}(N K)$ for batch $\mathrm{NG}$ (neglecting sorting of prototypes). Because of the discrete locations of prototypes, the probability that prototypes are assigned to the same location becomes nonzero. This effect should be avoided e.g. by means of adding random noise to the distances in every run or via an explicit collision prevention mechanism, as in [52 for the median SOM.

Similarly, median SOM can be derived from the cost function $\hat{E}_{\mathrm{SOM}}$. The following algorithm is obtained:

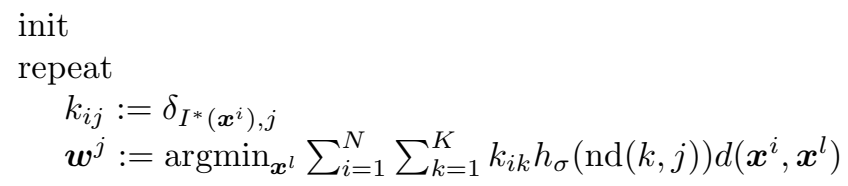


As for median NG, prototypes have to be determined by exhaustive search leading to $\mathcal{O}\left(N^{2} K\right)$ complexity per epoch.

Median clustering can be used for any given matrix $D$. It has been shown in 13. that both, median SOM and median NG converge in a finite number of steps, because both methods subsequently minimize the respective underlying cost function until they arrive at a fixed point of the algorithm. A simple demonstration of the behavior of median NG can be found in Fig. 1. The popular iris data set (see [15:5]) consists of 150 points and 3 classes.

Data are standardized to z-scores and batch NG and median NG is applied using 6 prototypes. Dimensions 3 and 4 are plotted together with the prototypes found by batch NG and median NG, respectively in Fig. 11. Obviously, the result is very similar, the quantization error of batch NG being 40.96, while median NG displays the slightly larger quantization error 44.85 due to the restriction of prototypes to data points. The classification accuracy obtained by posterior labeling is 0.84 for batch NG as compared to 0.92 for median NG. Since the prior class information is not taken into account during training, standard batch NG yields a larger classification error despite from its larger prototype flexibility.

\section{Supervision}

Often, additional label information is available for (parts of) the data set. Unsupervised data inspection and clustering does not take this information into account and cluster boundaries do not follow class distributions of the priorly known classes unless they coincide with unsupervised clusters in the data. This can be avoided by taking prior label information into account. We assume that a class label $\boldsymbol{y}^{i}$ is available for every data point $\boldsymbol{x}^{i}$. We assume $\boldsymbol{y}^{i} \in \mathbb{R}^{d}$, i.e. $d$ classes are represented by means of a full disjunctive coding (i.e., $y_{j}^{i}=\delta_{c^{i}, j}$, where $c^{i}$ is the index of the class for the data point $\boldsymbol{x}^{i}$ ), including the possibility of fuzzy assignments. We equip every prototype $\boldsymbol{w}^{j}$ with a label $\boldsymbol{Y}^{j} \in \mathbb{R}^{d}$ which is adapted during training. This vector represents the class label of the prototype, i.e. the average labels of data in its receptive field.

The aim of semisupervised clustering and data inspection is to determine prototypes and their labels in such a way that prototypes represent data point faithfully and they take the labeling into account, i.e. the prototype labels should correspond to the label of data points of its receptive field. To achieve this goal, the distance of a prototype $\boldsymbol{w}^{j}$ from a data point $\boldsymbol{x}^{i}$ is extended towards

$$
d_{\beta}\left(\boldsymbol{x}^{i}, \boldsymbol{w}^{j}\right):=\beta \cdot d\left(\boldsymbol{x}^{i}, \boldsymbol{w}^{j}\right)+(1-\beta) \cdot d\left(\boldsymbol{y}^{i}, \boldsymbol{Y}^{j}\right)
$$

where $d\left(\boldsymbol{y}^{i}, \boldsymbol{Y}^{j}\right)$ denotes the squared Euclidean distance of the labels $\boldsymbol{y}^{i}$ and $\boldsymbol{Y}^{j}$, and $\beta \in(0,1)$ balances the goal to represent the input data and the labels within the receptive field correctly.

This extended distance measure can be directly integrated into the cost functions of NG and SOM. Depending on the form of the distance measure, an extension of batch optimization or median optimization becomes possible. In 
both cases, the standard batch optimization scheme is accompanied by the label updates, which yields

$$
\boldsymbol{Y}^{j}=\sum_{i} h_{\sigma}\left(k_{i j}\right) \cdot y^{i} / \sum_{i} h_{\sigma}\left(k_{i j}\right)
$$

for batch and median NG, respectively, and

$$
\boldsymbol{Y}^{j}=\sum_{i k} k_{i k} h_{\sigma}(\operatorname{nd}(k, j)) \cdot y^{i} / \sum_{i k} k_{i k} h_{\sigma}(\operatorname{nd}(k, j))
$$

for batch and median SOM, respectively. It can be shown in the same way as for standard batch and median clustering, that these supervised variants converge after a finite number of epochs towards a fixed point of the algorithm.

The effect of supervision can exemplarly be observed for the iris data set: supervised batch NG with supervision parameter $\beta=0.5$ causes the prototypes to follow more closely the prior class borders in particular in overlapping regions and, correspondingly, an improved classification accuracy of 0.95 is obtained for supervised batch NG. The mixture parameter $\beta$ constitutes a hyperparameter of training which has to be optimized according to the given data set. However, in general the sensitivity with respect to $\beta$ seems to be quite low and the default value $\beta=0.5$ is often a good choice for training. Further, supervision for only part of the training data $\boldsymbol{x}^{i}$ is obviously possible.

\section{Experiments}

We demonstrate the behavior of median NG for a variety of biomedical benchmark problems. NG is intended for unsupervised data inspection, i.e. it can give hints on characteristic clusters and neighborhood relationships in large data sets. For the benchmark examples, prior class information is available, such that we can evaluate the methods by means of their classification accuracy. For semisupervised learning, prototypes and corresponding class labels are directly obtained from the learning algorithm. For unsupervised training, posterior labeling of the prototypes based on a majority vote of their receptive fields can be used. For all experiments, repeated cross-validation has been used for the evaluation.

Wisconsin breast cancer The Wisconsin breast cancer diagnostic database is a standard benchmark set from clinical proteomics [56]. It consists of 569 data points described by 30 real-valued input features: digitized images of a fine needle aspirate of breast mass are described by characteristics such as form and texture of the cell nuclei present in the image. Data are labeled by two classes, benign and malignant.

The data set is contained in the Euclidean space such that we can compare all clustering versions as introduced above for this data set using the Euclidean metric. We train 40 neurons using 200 epochs. The dataset is standardized to z-scores and randomly split into two halfs for each run. The result on the test set 
averaged over 100 runs is reported. We obtain a test set accuracy of 0.957 for the supervised version and 0.935 for the unsupervised version, both setting $\beta=0.1$ which is optimum for these cases. Results for simple K-means without neighborhood cooperation yield an accuracy 0.938 for standard (unsupervised) K-means resp. 0.941 for supervised K-means. Obviously, there are only minor, though significant differences of the results of the different clustering variants on this data set: incorporation of neighborhood cooperation allows to improve K-means, incorporation of label information allows to improve fully unsupervised clustering. As expected, Euclidean clustering is superior to median versions (using the squared Euclidean norm) because the number of possible prototype locations is reduced for median clustering. However, the difference is only $1.3 \%$, which is quite remarkable because of the comparably small data set, thus dramatically reduced flexibility of prototype locations.

The article 56 reports a test set accuracy of $0.97 \%$ using 10 -fold crossvalidation and a supervised learning algorithm (a large margin linear classifier including feature selection). This differs from our best classification result by $1.8 \%$. Thereby, the goal of our approach is a faithful prototype-based representation of data, such that the result is remarkable.

Chromosomes The Copenhagen chromosomes database is a benchmark from cytogenetics [45]. A set of 4200 human chromosomes from 22 classes (the autosomal chromosomes) are represented by the grey levels of their images and transferred to strings which represent the profile of the chromosome by the thickness of their silhouettes. This data set is non-Euclidean, consisting of strings of different length, and standard neural clustering cannot be used. Median versions, however, are directly applicable. The edit distance (also known as the Levenshtein distance [43]), is a typical distance measure for two strings of different length, as described in 35,49. In our application, distances of two strings are computed using the standard edit distance whereby substitution costs are given by the signed difference of the entries and insertion/deletion costs are given by 4.5 [49].

The algorithms have been run using 100 neurons and 100 epochs per run. Supervised median neural gas achieves an accuracy of 0.89 for $\beta=0.1$. This improves by $6 \%$ compared to median K-means. A larger number of prototypes allows to further improve this result: 500 neurons yield an accuracy of 0.93 for supervised median neural gas clustering and 0.91 for supervised median K-means clustering, both taken for $\beta=0.1$. This is already close to the results of fully supervised k-nearest neighbor classification which uses all points of the training set for classification. 12-nearest neighbors with the standard edit distance yields an accuracy 0.944 as reported in [35] whereas more compact classifiers such as feedforward networks or hidden Markov models only achieve an accuracy less than 0.91 , quite close to our results for only 100 prototypes. 


\section{Proteins}

The evolutionary distance of 226 globin proteins is determined by alignment as described in 48]. These samples originate from different protein families: hemoglobin- $\alpha$, hemoglobin- $\beta$, myoglobin, etc. Here, we distinguish five classes as proposed in [23]: HA, HB, MY, GG/GP, and others.

We use 30 neurons and 300 epochs per run. The accuracy on the test set averaged over 50 runs is reported in Fig. 2. Here, optimum mixing parameters can be observed for supervised median neural gas and $\beta \in[0.5,0.9]$, indicating that the statistics of the inputs guides the way towards a good classification accuracy. However, an integration of the labels improves the accuracy by nearly $10 \%$ compared to fully unsupervised clustering. As beforehand, integration of neighborhood cooperation is well suited in this scenario. Unlike the results reported in 23 for SVM which uses one-versus-rest encoding, the classification in our setting is given by only one clustering model. Depending on the choice of the kernel, 23] reports errors which approximately add up to 0.04 for the leaveone-out error. This result, however, is not comparable to our results due to the different error measure. A 1-nearest neighbor classifier yields an accuracy 0.91 for our setting ( $\mathrm{k}$-nearest neighbor for larger $\mathrm{k}$ is worse; 23] reports an accumulated leave-one-out error of 0.065 for 1-nearest neighbor) which is comparable to our (clustering) results.

Thus, unsupervised or semi-supervised data inspection which accounts for both, data statistics and prior labeling, reaches a classification accuracy comparable to fully supervised approaches, i.e. the clusters found by median NG are meaningful in these cases.

\section{Fast implementations}

As pointed out previously, the computational cost of median clustering algorithms is quite high: the exhaustive search for the best prototypes leads to a cost of $\mathcal{O}\left(N^{2} K\right)$ per epoch (for both median NG and median SOM). This cost is induced by the need for evaluating a sum of the following form

$$
\sum_{i=1}^{N} \alpha_{i, l, j} d\left(\boldsymbol{x}^{i}, \boldsymbol{x}^{l}\right)
$$

where $\boldsymbol{x}^{l}$ is a candidate for prototype $j$. Evaluating this sum is a $\mathcal{O}(N)$ operation which has to be repeated for each candidate $(N$ possibilities $)$ and for each prototype ( $K$ times). As the coefficients of the sums depends on $j$, it might seem at first glance that there is no way to reduce the cost.

\subsection{Block summing}

However, the prior structure of the SOM can be leveraged to reduce the total cost to $\mathcal{O}\left(N^{2}+N K^{2}\right)$ per epoch 12 . Let $C_{j}^{*}$ denote the receptive field of prototype 
$j$, more precisely

$$
C_{j}^{*}:=\left\{i \in\{1, \ldots, N\} \mid I^{*}\left(\boldsymbol{x}^{i}\right)=j\right\}
$$

Then the prototype $\boldsymbol{w}^{j}$ is given by

$$
\boldsymbol{w}^{j}=\arg \min _{\boldsymbol{x}^{l}} \sum_{k=1}^{K} h_{\sigma}(\operatorname{nd}(k, j)) \sum_{i \in C_{k}^{*}} d\left(\boldsymbol{x}^{i}, \boldsymbol{x}^{l}\right) .
$$

The main advantage of this formulation over the standard one is that there is now a clean separation between components that depend on $j$ (the $h_{\sigma}(\operatorname{nd}(k, j))$ terms) and those that do not (the sums $\left.\sum_{i \in C_{k}^{*}} d\left(\boldsymbol{x}^{i}, \boldsymbol{x}^{l}\right)\right)$. This leads to the following version of the median SOM 12 :

$$
\begin{aligned}
& \text { init } \\
& \text { repeat } \\
& \qquad C_{j}^{*}:=\left\{i \in\{1, \ldots, N\} \mid I^{*}\left(\boldsymbol{x}^{i}\right)=j\right\} \text { (receptive field calculation) } \\
& \quad S(k, l):=\sum_{i \in C_{k}^{*}} d\left(\boldsymbol{x}^{i}, \boldsymbol{x}^{l}\right)(\text { block summing) } \\
& \quad \boldsymbol{w}^{j}:=\arg \min _{\boldsymbol{x}^{l}} \sum_{k=1}^{K} h_{\sigma}(\operatorname{nd}(k, j)) S(k, l) \text { (prototype calculation) }
\end{aligned}
$$

There are $N \times K$ block sums $S(k, l)$ which can be computed in $\mathcal{O}\left(N^{2}\right)$ operations as the $\left(C_{k}^{*}\right)_{1 \leq k \leq N}$ form a partition of the dataset. Then the exhaustive search involves only summing $K$ values per candidate prototype (and per prototype), leading to a total cost of $\mathcal{O}\left(N K^{2}\right)$ (of the same order as the computation of the receptive fields). The total computational load is therefore $\mathcal{O}\left(N^{2}+N K^{2}\right)$. In practice, the speed up is very high. For instance, with the optimized Java implementation proposed in 12$]^{3}$, a standard $\mathcal{O}\left(N^{2} K\right)$ implementation of the median SOM uses approximately 5.7 seconds per epoch on the Chromosomes dataset $(N=4200)$ for $K=100$ prototypes (arranged on a $10 \times 10$ hexagonal grid) on a standard workstation 1 . Under identical conditions, the above algorithm uses only 0.25 second per epoch while providing exactly the same results (this is 23 times faster than the standard implementation, see Table 1 for a summary of the timing results for the Median SOM variants).

Obviously, the speed up strongly depends on both $N$ and $K$. For example if $K$ is raised to 484 (for a regular hexagonal grid of size $22 \times 22$ ), the standard implementation of the median SOM uses approximately 30.1 seconds per epoch, with the block summing algorithm uses only 3.67 seconds per epoch. This is still more than 8 times faster than the standard implementation, but as expected the speed up factor is worse than with a lower value of $K$. Nevertheless as reflected in the theoretical cost analysis, extensive simulations conducted in [12] have shown that the block summing algorithm is always faster than the standard approach which has therefore no reason to be used.

\footnotetext{
${ }^{3}$ Available at http://gforge.inria.fr/projects/somlib/

${ }^{4}$ AMD Athlon $643000+$ processor with 2GB of main memory, running Fedora Linux 7 and with Sun 1.6 java virtual machine in server mode
} 


\subsection{Heuristic search}

Additional reduction in the actual running time of the median SOM can be obtained via the branch and bound principle from combinatorial optimization [11. The goal of branch and bound 42] is to avoid to perform an exhaustive search to solve a minimization problem by means of two helper procedures. The first procedure is a partition method to be applied to the search space (the branch part of the method). The second procedure provides quickly a guaranteed lower bound of the criterion to be minimized on any class of the partition of the search space (the bound part of the method). By quickly one means faster than an exhaustive evaluation of the criterion on the class.

A standard implementation of the minimization of a function $f$ by an exhaustive search on the search space $\mathcal{S}$ proceeds as follows:

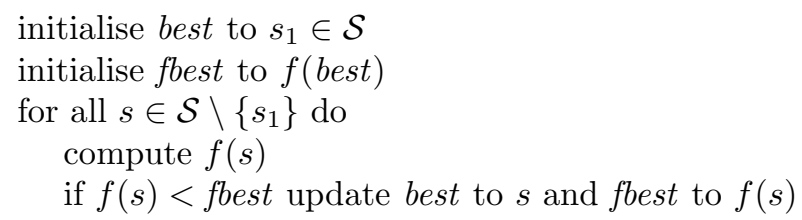

To save some evaluations of $f$, a branch and bound search proceeds as follows:

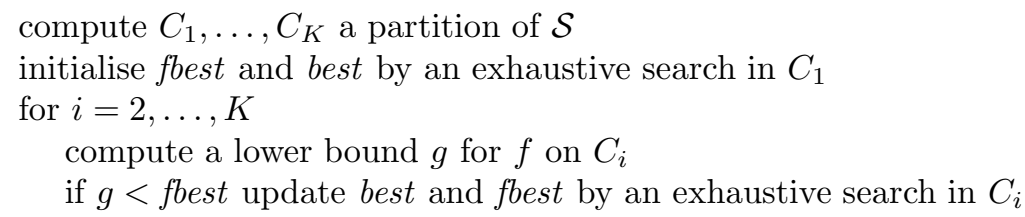

The gain comes from the possibility of pruning entire regions (classes) of the search space when the lower bound of the criterion $f$ on such a region is higher than the best value found so far. The best gain is achieved when all regions except $C_{1}$ are pruned. Obviously, the order in which the partition classes are searched is crucial in obtaining good performances.

In the median SOM, the search space is the dataset. This provides a natural branching procedure as the receptive fields $\left(C_{j}^{*}\right)_{1 \leq j \leq K}$ of the prototypes of the SOM build a partition of the dataset. If the receptive fields have comparable sizes, i.e., around $N / K$, branch and bound can reduce the search space for each prototype from a size of $N$ to a size of $N / K$, in the optimal case (perfect branching). This could reduce the cost of the search from $\mathcal{O}\left(N K^{2}\right)$ to $\mathcal{O}\left(N K+K^{2}\right)$. Indeed, in the ideal case, one would only evaluate the quality criterion $\sum_{k=1}^{K} h_{\sigma}(\operatorname{nd}(k, j)) S(k, l)$ for candidate prototypes from one cluster (this would cost $\mathcal{O}(K(N / K))=\mathcal{O}(N))$ and then compare the best value to the lower bound of each other cluster $(\mathcal{O}(K)$ additional operations).

The bounding procedure needs to provide a tight lower bound for the following quantity

$$
\min _{\boldsymbol{x}^{l} \in C_{m}^{*}} \sum_{k=1}^{K} h_{\sigma}(\operatorname{nd}(k, j)) S(k, l) \text {. }
$$


A class of lower bounds is given by the following equation

$$
\eta(m, j, \Theta):=\sum_{k \in \Theta} h_{\sigma}(\operatorname{nd}(k, j)) \min _{\boldsymbol{x}^{l} \in C_{m}^{*}} S(k, l),
$$

where $\Theta$ is a subset of $\{1, \ldots, K\}$. There are several reasons for using such bounds. First the quantity $\min _{\boldsymbol{x}^{l} \in C_{m}^{*}} S(k, l)$ depends only on $k$ and $l$ : it can be computed once and for all before the exhaustive search phase (in fact in parallel with the computation of the block sum $S(k, l)$ itself). The additional cost is negligible compared to other costs (there are $K^{2}$ values which are computed in $\mathcal{O}(N K)$ operations). However, computing $\eta(m, j,\{1, \ldots, K\})$ is costly, as the search process will need this bound for all $m$ and $j$, leading to a total cost of $\mathcal{O}\left(K^{3}\right)$ : this is small compared to $\mathcal{O}\left(N^{2}+N K^{2}\right)$ but not negligible when $K$ is large, especially compared to the best case cost $\left(\mathcal{O}\left(N^{2}+N K+K^{2}\right)\right.$ with perfect branching).

It is therefore interesting in theory to consider strict subsets of $\{1, \ldots, K\}$, in particular the singleton $\Theta=\{j\}$ which leads to the very conservative lower bound $h_{\sigma}(\operatorname{nd}(j, j)) \min _{\boldsymbol{x}^{l} \in C_{m}^{*}} S(j, l)$, for which the computation cost is only $\mathcal{O}\left(K^{2}\right)$ for all $m$ and $j$. Despite its simplicity, this bound leads to very good results in practice [11] because when the neighborhood influence is annealed during training, $h_{\sigma}(\operatorname{nd}(k, j))$ gets closer and closer to the Kronecker function $\delta_{k, j}$.

Compared to the improvements generated by reducing the complexity to $\mathcal{O}\left(N^{2}+N K^{2}\right)$, the speed up provided by branch and bound is small. Under exactly the same conditions as in the previous section, the time needed per epoch is 0.22 second (compared to 0.25 ) when the bounds are computed with $\Theta=\{j\}$ and 0.14 second when $\Theta=\{1, \ldots, K\}$ (which shows that perfect branching does not happen as the $\mathcal{O}\left(K^{3}\right)$ cost of the bounds calculation does not prevent from getting a reasonable speed up). Complex additional programming tricks exposed in [11, 12] can reduce even further the running time in some situation (e.g., when $K$ is very large), but on the Chromosomes dataset with $K=100$, the best time is obtained with the algorithm described above. The speed up compared to a naive implementation is nevertheless quite large as the training time is divided by 40 , while the results are guaranteed to be exactly identical.

\begin{tabular}{lcc} 
Algorithm & $K=100$ & $K=484$ \\
\hline Standard implementation & 5.74 & 30.1 \\
Block Summing & 0.248 & 3.67 \\
Branch and bound $\Theta=\{j\}$ & 0.224 & 2.92 \\
Branch and bound $\Theta=\{1, \ldots, K\}$ & 0.143 & 1.23 \\
Branch and bound $\Theta=\{1, \ldots, K\}$ and early stopping & 0.141 & 0.933 \\
\hline
\end{tabular}

Table 1. Average time needed to complete an epoch of the Median SOM (in seconds) for the Chromosomes dataset

When $K$ is increased to 484 as in the previous section, the time per epoch for $\Theta=\{j\}$ is 2.92 seconds and 1.23 seconds when $\Theta=\{1, \ldots, K\}$. In this case, 
the "early stopping" trick described in 11,12 (see also the next Section) can be used to bring down this time to 0.93 second per epoch. This is more than 32 times faster than the naive implementation and also almost 4 times faster than the block summing method. Branch and bound together with early stopping complement therefore the block summing improvement in the following sense: when $K$ is small (of the order of $\sqrt{N}$ or below), block summing is enough to reduce the algorithmic cost to an acceptable $\mathcal{O}\left(N^{2}\right)$ cost. While $K$ grows above this limit, branch and bound and early stopping manage to reduce the influence of the $\mathcal{O}\left(N K^{2}\right)$ term on the total running time.

\subsection{Median Neural Gas}

Unfortunately the solutions described above cannot be applied to median NG, as there is no way to factor the computation of $\sum_{i=1}^{N} h_{\sigma}\left(k_{i j}\right) d\left(\boldsymbol{x}^{i}, \boldsymbol{x}^{l}\right)$ in a similar way as the one used by the median SOM. Indeed, the idea is to find in the sum sub-parts that do not depend on $j$ (the prototype) so as to re-use them for all the prototypes. The only way to achieve this goal is to use a partition on the dataset (i.e., on the index $i$ ) such that the values that depend on $j, h_{\sigma}\left(k_{i j}\right)$, remain constant on each class. This leads to the introduction of a partition $R^{j}$ whose classes are defined by

$$
R_{k}^{j}=\left\{i \in\{1, \ldots, N\} \mid \operatorname{rk}\left(\boldsymbol{x}^{i}, \boldsymbol{w}^{j}\right)=k\right\} .
$$

This is in fact the partition induced by the equivalence relation on $\{1, \ldots, N\}$ defined by $i \sim_{j} i^{\prime}$ if and only if $h_{\sigma}\left(k_{i j}\right)=h_{\sigma}\left(k_{i^{\prime} j}\right)$. Using this partition, we have

$$
\sum_{i=1}^{N} h_{\sigma}\left(k_{i j}\right) d\left(\boldsymbol{x}^{i}, \boldsymbol{x}^{l}\right)=\sum_{k=1}^{K} h_{\sigma}(k) \sum_{i \in R_{k}^{j}} d\left(\boldsymbol{x}^{i}, \boldsymbol{x}^{l}\right) .
$$

At first glance, this might look identical to the factorisation used for the median SOM. However there is a crucial difference: here the partition depends on $j$ and therefore the block sums $\sum_{i \in R_{k}^{j}} d\left(\boldsymbol{x}^{i}, \boldsymbol{x}^{l}\right)$ cannot be precomputed (this factorization will nevertheless prove very useful for early stopping).

For both algorithms, the fast decrease of $h_{\sigma}$ suggests an approximation in which small values of $\alpha_{i, l, j}$ (i.e., of $h_{\sigma}\left(k_{i j}\right)$ and of $h_{\sigma}(\operatorname{nd}(k, j))$ ) are discarded. After a few epochs, this saves a lot of calculation but the cost of initial epochs remains unchanged. Moreover, this approximation scheme changes the results of the algorithms, whereas the present section focuses on exact and yet fast implementation of the median methods.

A possible source of optimization for median NG lies in the so called "early stopping" strategy exposed for the median SOM in [11, 12]. The idea is to leverage the fact that the criterion to minimize is obtained by summing positive values. If the sum is arranged in such as way that large values are added first, the partial result (i.e., the sum of the first terms of the criterion) can exceed the best value obtained so far (from another candidate). Then the loop that implements the 
summing can be stopped prematurely reducing the cost of the evaluation of the criterion. Intuitively, this technique works if the calculation are correctly ordered: individual values in the sum should be processed in decreasing order while candidate prototypes should be tested in order of decreasing quality.

For the median SOM, as shown in [11,12], early stopping, while interesting in theory, provides speed up only when $K$ is large as the use of block summing has already reduced the cost of the criterion evaluation from $\mathcal{O}(N)$ to $\mathcal{O}(K)$. On the Chromosomes dataset for instance, the previous Section showed that early stopping gains nothing for $K=100$ and saves approximately $24 \%$ of the running time for $K=484$ (see Table 1).

However, as there is no simplification in the criterion for median NG, early stopping could cause some improvement, especially as the sorting needed to compute the rk function suggests an evaluation order for the criterion and an exploration order during the exhaustive search.

The computation of $\boldsymbol{w}^{j}:=\arg \min _{x^{l}} \sum_{i=1}^{N} h_{\sigma}\left(k_{i j}\right) d\left(\boldsymbol{x}^{i}, \boldsymbol{x}^{l}\right)$ by an early stopping algorithm takes the following generic form:

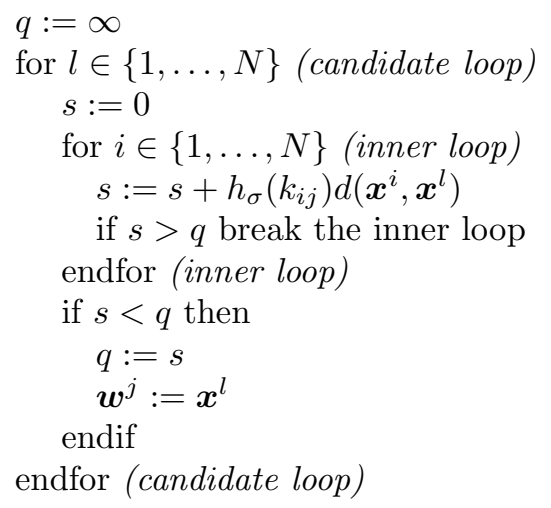

Ordering Both loops (candidate and inner) can be performed in specific orders. The candidate loop should analyse the data points $\boldsymbol{x}^{l}$ in decreasing quality order, i.e., it should start with $\boldsymbol{x}^{l}$ such that $\sum_{i=1}^{N} h_{\sigma}\left(k_{i j}\right) d\left(\boldsymbol{x}^{i}, \boldsymbol{x}^{l}\right)$ is small and end with the points that have a large value of this criterion. This optimal order is obviously out of reach because computing it implies the evaluation of all the values of the criterion, precisely what we want to avoid. However, Median Neural Gas produces clusters of similar objects, therefore the best candidates for prototype $\boldsymbol{w}^{j}$ are likely to belong to the receptive field of this prototype at the previous epoch. A natural ordering consists therefore in trying first the elements of this receptive field and then elements of the receptive fields of other prototypes in increasing order of dissimilarities between prototypes. More precisely, with

$$
C_{k}:=\left\{i \in\{1, \ldots, N\} \mid I\left(\boldsymbol{x}^{i}\right)=k\right\},
$$

the candidate loop starts with $C_{j_{1}}=C_{j}$ and proceeds through the $\left(C_{j_{i}}\right)_{2 \leq i \leq K}$ with $d\left(\boldsymbol{w}^{j}, \boldsymbol{w}^{j_{i-1}}\right) \leq d\left(\boldsymbol{w}^{j}, \boldsymbol{w}^{j_{i}}\right)$. Computing this order is fast $\left(\mathcal{O}\left(K^{2} \log K\right)\right.$ operations for the complete prototype calculation step). 
Another solution consists in ordering the candidate loop according to increasing ranks $k_{l j}$ using the partition $R^{j}$ defined previously instead of the receptive fields.

The inner loop should be ordered such that $s$ increases as quickly as possible: high values of $h_{\sigma}\left(k_{i j}\right) d\left(\boldsymbol{x}^{i}, \boldsymbol{x}^{l}\right)$ should be added first. As for the candidate loop this cannot be achieved exactly without loosing all benefits of the early stopping. A simple solution consists again in using the partition $R^{j}$. The rational is that the value of $h_{\sigma}\left(k_{i j}\right) d\left(\boldsymbol{x}^{i}, \boldsymbol{x}^{l}\right)$ is likely to be dominated by the topological term $h_{\sigma}\left(k_{i j}\right)$, especially after a few epochs when $h_{\sigma}$ becomes peaked in 0 . This ordering has three additional benefits. As explained above, the factorized representation provided by equation (19) allows to save some calculations. Moreover, $R^{j}$ can be computed very efficiently, as a side effect of computing the ranks $k_{i j}$. If they are stored in a $N K$ array, $R^{j}$ is obtained by a single pass on the index set $\{1, \ldots, N\}$. Computing the $R^{j}$ for all $j$ has therefore a $\mathcal{O}(N K)$ cost. Finally, $R^{j}$ can also be used for ordering the candidate loop.

It should be noted that using different orderings for the candidate loop can lead to different final results for the algorithm in case of ties between candidates for prototypes. In practice, the influence of such differences is extremely small, but contrarily to the SOM for which all experiments produced in 11,12] and in this chapter gave exactly the same results, regardless of the actual implementation, variants of the Median Neural Gas exhibit a small variability in the results (less than one percent of differences in the quantization error, for instance). Those differences have been neglected as they could be suppressed via a slightly more complex implementation in which ties between candidate prototypes are broken at random (rather than via the ordering); using the same random generator would produce exactly the same results in all implementations.

Early stopping granularity Experiments conducted in [11.12 have shown that early stopping introduces a non negligible overhead to the inner loop simply because it is the most intensive part of the algorithm which is executed $N^{2} K$ times in the worst case. A coarse grain early stopping strategy can be used to reduce the overhead at the price of a more complex code and of less early stops. The idea is to replace the standard inner loop by the following version:

$$
\begin{aligned}
& s:=0 \\
& \text { for } m \in\{1, \ldots, M\} \text { (monitoring loop) } \\
& \quad \text { for } i \in B_{m} \text { (internal loop) } \\
& \quad s:=s+h_{\sigma}\left(k_{i j}\right) d\left(\boldsymbol{x}^{i}, \boldsymbol{x}^{l}\right) \\
& \quad \text { endfor (internal loop) } \\
& \quad \text { if } s>q \text { break the monitoring loop } \\
& \text { endfor (monitoring loop) }
\end{aligned}
$$

The main device is a partition $B=\left(B_{1}, \ldots, B_{M}\right)$ of $\{1, \ldots, N\}$ which is used to divide the computation into uninterrupted calculations (internal loops) and to check on a periodic basis by the monitoring loop. The value of $M$ can be used to tune the grain of the early stopping with a classical trade-off between the 
granularity of the monitoring and its overhead. In this chapter, we have focused on a particular way of implementing this idea: rather than using an arbitrary partition $B$, we used the $R^{j}$ partition. It has two advantages over an arbitrary one: is provides an interesting ordering of the monitoring loop (i.e., in decreasing order of $\left.h_{\sigma}\left(k_{i j}\right)\right)$ and allows the code to use the factorized equation (19).

\begin{tabular}{lcc} 
Algorithm & $K=100$ & $K=500$ \\
\hline Standard implementation & 5.31 & 25.9 \\
Early stopping without ordering & 5.26 & 24.3 \\
Early stopping with candidate loop ordering & 4.38 & 20.7 \\
Full ordering and fine grain early stopping & 1.16 & 7.45 \\
Full ordering and coarse grain early stopping & 0.966 & 5.91
\end{tabular}

Table 2. Average time needed to complete an epoch of the Median Neural Gas (in seconds) for the Chromosomes dataset

Experiments Variants of the early stopping principle applied to Median Neural Gas were tested on the Chromosomes $(N=4200)$ with $K=100$ and $K=500$. They are summarized in Table 2. The standard implementation corresponds to the exhaustive search $\mathcal{O}\left(N^{2} K\right)$ algorithm. The need for ordering is demonstrated by the results obtained by a basic implementation of early stopping in which the natural data ordering is used for both candidate and inner loops. Moreover, while the candidate loop order based on receptive fields reduces the running time, the gain remains limited when the inner loop is not ordered (the running time is reduced by approximately $20 \%$ compared to the standard implementation).

Much better improvements are reached when the $R^{j}$ partitions are used to order both loops. The running time is divided by more than 5 for $K=100$ and by more than 4 for $K=500$, when a coarse grain early stopping method is used. The fine grain version is slightly less efficient because of the increased overhead in the inner loop.

The structure of the Median Neural Gas algorithm prevents the use of the block summing trick which is the main source of improvement for the Median SOM. In the case of Neural Gas, early stopping provides better improvement over the state-of-the-art implementation, than it does for the SOM, because it targets an internal loop with $\mathcal{O}(N)$ complexity whereas the block summing approach leads to a $\mathcal{O}(K)$ inner loop. In the end, the optimized Median SOM remains much faster than the Median Neural Gas (by a factor 6). However, the SOM is also very sensitive to its initial configuration whereas Neural Gas is rather immune to this problem. In practice, it is quite common to restart the SOM several times from different initial configuration, leading to quite comparable running time for both methods. 


\section{Approximate patch clustering for large data sets}

A common challenge today [57], arising especially in computational biology, image processing, and physics, are huge datasets whose pairwise dissimilarities cannot be hold at once within random-access memory during computation, due to the sheer amount of data (a standard workstation with 4 GB of main memory cannot hold more than $N=2^{15}$ data points when they are described by a symmetric dissimilarity matrix). Thus, data access is costly and only a few, ideally at most one pass through the data set is still affordable.

Most work in this area can be found in the context of heuristic (possibly hierarchical) clustering on the one side and classical K-means clustering on the other side. Heuristic algorithms often directly assign data consecutively to clusters based on the distance within the cluster and allocate new clusters as required. Several popular methods include CURE, STING, and BIRCH [22,55,58]. These methods do not rely on a cost function such that an incorporation of label information into the clustering becomes difficult.

Extensions of K-means clustering can be distinguished into methods which provide guarantees on the maximum difference of the result from classical Kmeans, such as presented in the approaches 21,34]. However, these variants use resources which scale in the worst case with a factor depending on $N$ ( $N$ being the number of points) with respect to memory requirements or passes through the data set. Alternatives are offered by variants of K-means which do not provide approximation guarantees, but which can be strictly limited with respect to space requirements and time. An early approach has been proposed in [50]: data are clustered consecutively in small patches, whereby the characteristics of the data and the possibility to compress subsets of data are taken into account. A simpler although almost as efficient method has been proposed in 14: Standard K-means is performed consecutively for patches of the data whereby each new patch is enriched by the prototypes obtained in the previous patch. A sufficient statistics of the outcome of the last run can thereby easily be updated in a consecutive way, such that the algorithm provides cluster centres after only one pass through the data set, thereby processing the data consecutively in patches of predefined fixed size.

Some of these ideas have been transferred to topographic maps: the original median SOM 41 proposes simple sampling to achieve efficient results for huge data sets. Simple sampling is not guaranteed to preserve the statistics of the data and some data points might not be used for training at all, because of which reason [41] proposes to use all data in the last run. An approach which uses all available statistics consists in an extension of patch clustering towards neural gas and alternatives, as proposed in [3.2]. This method processes data in patches, thereby integrating the sufficient statistics of results of the previous run, such that all available information processed so far is used in each consecutive clustering step. Since the runs rely on a statistics of the data, the overall result only approximates the optimum solution obtained by standard batch clustering. However, in practice, results are quite good. 
An extension of this method to median clustering is possible and yields promising results, as proposed in 29. Here we give an introduction to this simple and powerful extension of median clustering to huge data sets. As a side effect, this method dramatically reduces the complexity of clustering to linear complexity in $N, N$ being the number of data points.

Assume, as before, a dissimilarity matrix $D$ with entries $d_{i j}$ representing the dissimilarity of patterns. Here we assume symmetry of the matrix, but no further requirements need to be fulfilled. For huge data sets, only parts of the matrix $D$ fit into main memory. We assume that access to single elements of the matrix is possible at any time, e.g. the elements are stored in a database or the dissimilarity values are computed on the fly by means of some (possibly complex) dissimilarity measure (such as pairwise alignment of proteins using FASTA). The exact way how dissimilarities are accessed is not relevant for patch clustering.

During processing of patch Median NG, $n_{p}$ disjoint patches of fixed size $p=\left\lfloor m / n_{p}\right\rfloor$ are taken from the dissimilarity matrix $D$ consecutively 5 where every patch

$$
P_{i}=\left(d_{s t}\right)_{s, t=(i-1) \cdot p, \ldots, i \cdot p-1} \in \mathbb{R}^{p \times p}
$$

is a submatrix of $D$, representing data points $(i-1) \cdot p$ to $i \cdot p-1$. The patches are small such that they fit into main memory. The idea of the patch scheme is to add the prototypes from the processing of the former patch $P_{i-1}$ as additional datapoints to the current patch $P_{i}$, forming an extended patch $P_{i}^{*}$ to work on further. The additional datapoints - the former prototypes - are weighted according to the size of their receptive fields, i.e. how many datapoints they have been representing in the former patch. Therefore, every datapoint $\boldsymbol{x}^{i}$, as a potential prototype, is equipped with a multiplicity $m_{i}$, that is at first initialized with $m_{i}=1$. Unlike simple sampling strategies, every point of the dataset is considered exactly once and a sufficient statistics of all already processed data is passed to further patches by means of the weighted prototypes.

Unlike the situation of patch NG in Euclidean space [3,2], where inter-patch distances can always be recalculated with help of the Euclidean metric, we are now dealing with an unknown mathematical space. We have to construct the extended patch from given dissimilarity data. The extended patch $P_{i}^{*}$ is defined as

$$
P_{i}^{*}=\left(\begin{array}{c|c}
d\left(N_{i-1}\right) & d\left(N_{i-1}, P_{i}\right) \\
\hline d\left(N_{i-1}, P_{i}\right)^{T} & P_{i}
\end{array}\right)
$$

\footnotetext{
${ }^{5}$ The remainder is no further considered here for simplicity. In the practical implementation the remaining datapoints are simply distributed over the first $\left(M-p \cdot n_{p}\right)$ patches.
} 
where

$$
\begin{aligned}
& d\left(N_{i-1}\right)=\left(d_{s t}\right)_{s, t \in N_{i-1}} \in \mathbb{R}^{K \times K} \\
& d\left(N_{i-1}, P_{i}\right)=\left(d_{s t}\right)_{s \in N_{i-1}, t=(i-1) \cdot p, \ldots, i \cdot p-1} \in \mathbb{R}^{K \times p}
\end{aligned}
$$

denote the inter-distances of former prototypes and the distances between former prototypes and current patch points, respectively. Every point is weighted with a multiplicity $m_{j}$ which is set to 1 for all new points $j \in[(i-1) \cdot p, i \cdot p-1]$. For points which stem from prototypes, the multiplicity is set to the sum of the multiplicities of all points in its receptive field.

To apply median clustering, we have to incorporate these multiplicities into the learning scheme. The cost function becomes

$$
\hat{E}_{\mathrm{NG}} \sim \frac{1}{2 N} \cdot \sum_{i=1}^{N} \sum_{j=1}^{K} h_{\sigma}\left(\operatorname{rk}\left(\boldsymbol{x}^{i}, \boldsymbol{w}^{j}\right)\right) \cdot m_{j} \cdot d\left(\boldsymbol{x}^{i}, \boldsymbol{w}^{j}\right)
$$

where, as before, prototype locations $\boldsymbol{w}^{j}$ are restricted to data points. Optimum ranks are obtained as beforehand. Optimum prototypes are determined by means of the formula

$$
\boldsymbol{w}^{j}=\operatorname{argmin}_{\boldsymbol{x}^{l}} \sum_{i=1}^{N} h_{\sigma}\left(r_{i j}\right) \cdot m_{j} \cdot d\left(\boldsymbol{x}^{i}, \boldsymbol{x}^{l}\right)
$$

Picking up the pieces, we obtain the following algorithm:

\section{Patch Median Neural Gas}

Cut the first Patch $P_{1}$

Apply Median NG on $P_{1} \longrightarrow$ Prototypes $N_{1}$

Update Multiplicities $m_{j}$

Repeat for $i=2, \ldots, n_{p}$

Cut patch $P_{i}$

Construct Extended Patch $P_{i}^{*}$ using $P_{i}$ and $N_{i-1}$

Apply modified Median NG with Multiplicities

$\longrightarrow$ Prototypes $N_{i}$

Update Multiplicities $m_{j}$

Return final Prototypes $N_{n_{p}}$

Median SOM can be extended to patch clustering in a similar way.

We demonstrate the behavior of patch clustering on the breast cancer data set which has been used beforehand. Here, we compare data points with the Cosine Measure

$$
d_{\cos }\left(\boldsymbol{x}^{i}, \boldsymbol{x}^{j}\right)=1-\frac{\boldsymbol{x}^{i} \cdot \boldsymbol{x}^{j}}{\left\|\boldsymbol{x}^{i}\right\|_{2} \cdot\left\|\boldsymbol{x}^{j}\right\|_{2}} .
$$

Standard median batch NG for 40 neurons and 100 epochs yields an average classification accuracy of 0.95 in a repeated 10 -fold cross-validation. In comparison, patch median NG with 5 patches, i.e. 114 data points per patch, arrives at a 
classification accuracy of 0.94 , yielding only a slight decrease of the classification accuracy.

The complexity of standard median NG is improved by incorporation of patches, as can be seen as follows. Assume a fixed patch size $p$ independent of the number of datapoints, e.g. $p$ is chosen according to the main memory. Then the algorithm uses only $\mathcal{O}\left(\frac{m}{p} \cdot(p+K)^{2}\right)=\mathcal{O}(m \cdot p+m \cdot K)=\mathcal{O}(m)$ entries of the dissimilarity matrix, compared to $\mathcal{O}\left(\mathrm{m}^{2}\right)$ in the original Median NG method. Moreover, every epoch (within a patch) has complexity $\mathcal{O}\left(p^{2}\right)=$ constant as opposed to $\mathcal{O}\left(N^{2}\right)$ for an epoch in full median clustering. Therefore the method does not only overcome the problem of limited memory, it also dramatically accelerates the processing of datasets, what might be useful in time critical applications.

\section{Discussion}

Neural clustering methods such as SOM and NG offer robust and flexible tools for data inspection. In biomedical domains, data are often nonvectorial such that extensions of the original methods towards general dissimilarity data have to be used. In this chapter, we presented an overview about one particularly interesting technique which extends NG and SOM towards dissimilarities by means of the generalized median. Prototypes are restricted to data positions such that the standard cost functions are well defined and extensions such as supervision can easily be transferred to this setting. Moreover, this way, clusters are represented in terms of typical exemplars from the data set, i.e. the idea offers a data representation which can be easily interpreted by experts in biomedical domains.

These benefits are paid back by increased costs in a naive implementation of the algorithms, the complexity of one epoch being of order $N^{2}$ instead of $N$, where $N$ refers to the number of data points. Since data are represented by a general $N \times N$ dissimilarity matrix instead of $N$ single vectors, these increased costs are to some extent unavoidable if the full information contained in the data is considered. Nevertheless, a variety of structural aspects allow to reduce the costs of median clustering in practical situations.

We discussed a variety of techniques which lead to a dramatic decrease of the training time while (approximately) preserving the quality of the original methods. These approaches can be decomposed into exact methods which provably lead to the same results as the original implementation and approximations which slightly reduce the quality of the results in return for an improved efficiency. Exact methods include

- block summing for median SOM due to the specific and fixed structure of the SOM neighborhood; as pointed out in this chapter, block summing leads to a major reduction of the computation time in this case. The method cannot be applied to NG, though, because NG does not rely on a priorly fixed lattice structure. 
- branch and bound methods which allow to reduce the number of necessary computations depending on the situation at hand; usually, the computational savings strongly depend on the order in which computations are performed. As pointed out in this chapter, branching can be done with respect to candidate prototypes on the one hand and summands which contribute to the overall cost associated with one prototype on the other hand. For both settings, the topological ordering of the data suggests a natural decomposition of the whole search space into parts. This procedure yields to a significant reduction of the computational costs for NG in particular for later states of training with partially ordered setting. For SOM, the savings are only minor compared to savings by means of block summing, though possibly significant depending on the number of prototypes.

These methods lead to the same results as a naive implementation but run in a fraction of the time.

Compared to these approaches, approximate methods constitute a compromise of accuracy and complexity. We presented a patch clustering approach for median clustering, which processes data in patches of fixed size and integrates the results by means of the sufficient statistics of earlier runs. This way, the computation time is reduced from $\mathcal{O}\left(N^{2}\right)$ to $\mathcal{O}(N)$. In particular, only a small part of the dissimilarity matrix is considered in patch training. This has the additional benefit that, this way, only a finite and fixed memory size is required and the clustering method can readily be applied to huge streaming data sets. Further, since only a fraction of the dissimilarity matrix needs to be computed, this method is particularly suited for biomedical applications with complex dissimilarity measures such as alignment distance.

\section{References}

1. S. Al-Harbi and V. Rayward-Smith. The use of a supervised k-means algorithm on real-valued data with applications in health. In P.W.H.Chung, C.J.Hinde, and A.All, editors, IEA/AIE 2003, LNCS 2718, pages 575-581. 2003.

2. N. Alex and B. Hammer. Parallelizing single pass patch clustering. In M.Verleysen, editor, ESANN'2008, pages 227-232, 2008.

3. N. Alex, B. Hammer, and F. Klawonn. Single pass clustering for large data sets. In Proceedings of 6th International Workshop on Self-Organizing Maps (WSOM 2007), Bielefeld, Germany, September 3-6, 2007, 2007.

4. C. Ambroise and G. Govaert. Analyzing dissimilarity matrices via Kohonen maps. In Proceedings of 5th Conference of the International Federation of Classification Societies (IFCS 1996), volume 2, pages 96-99, Kobe (Japan), March 1996.

5. E. Anderson. The irises of the gaspe peninsula. Bulletin of the American Iris Society, 59:25, 1935.

6. S. Arora, P. Raghavan, and S. Rao. Approximation schemes for euclidean kmedians and related problems. In Proceedings of the 30th Annual ACM Symposium on Theory of Computing, pages 106-113, 1998.

7. G. Barreto. Time series prediction with the self-organizing map: A review. In B. Hammer and P. Hitzler, editors, Perspectives on Neural-Symbolic Integration. Springer, 2007. 
8. R. Boulet, B. Jouve, F. Rossi, and N. Villa. Batch kernel som and related laplacian methods for social network analysis. Neurocomputing, 2008. to be published.

9. G. Celeux, E. Diday, G. Govaert, Y. Lechevallier, and H. Ralambondrainy. Classification Automatique des Données. Bordas, Paris, 1989.

10. M. Charikar, S. Guha, A.Tardos, and D. Shmoys. A constant-factor approcimation algorithm for the k-median problem. Journal of Computer and System Sciences, 65:129, 2002.

11. B. Conan-Guez and F. Rossi. Speeding up the dissimilarity self-organizing maps by branch and bound. In A. Sandoval, F.and Prieto, J. Cabestany, and M. Graña, editors, Proceedings of 9th International Work-Conference on Artificial Neural Networks (IWANN 2007), volume 4507 of Lecture Notes in Computer Science, pages 203-210, San Sebastián (Spain), June 2007.

12. B. Conan-Guez, F. Rossi, and A. El Golli. Fast algorithm and implementation of dissimilarity self-organizing maps. Neural Networks, 19(6-7):855-863, July-August 2006.

13. M. Cottrell, B. Hammer, A. Hasenfuss, and T. Villmann. Batch and median neural gas. Neural Networks, 19:762-771, 2006.

14. F.Farnstrom, J.Lewis, and C.Elkan. Scalability for clustering algorithms revisited. SIGKDD Explorations, 2(1):51-57, 2000.

15. R. A. Fisher. The use of multiple measurements in axonomic problems. Annals of Eugenics, 7:179-188, 1936.

16. J.-C. Fort, P. Letrémy, and M. Cottrell. Advantages and drawbacks of the batch kohonen algorithm. In M. Verleysen, editor, ESANN'2002, pages 223-230. D Facto, 2002.

17. B. Frey and D. Dueck. Clustering by passing messages between data points. Science, 315:972-977, 2007.

18. B. Frey and D. Dueck. Response to 'clustering by passing messages between data points'. Science, 319:726d, 2008.

19. T. Graepel, R. Herbrich, P. Bollmann-Sdorra, and K. Obermayer. Classification on pairwise proximity data. In NIPS, vol. 11, pages 438-444. MIT Press, 1999.

20. T. Graepel and K. Obermayer. A stochastic self-organizing map for proximity data. Neural Computation, 11:139-155, 1999.

21. S. Guha, N. Mishra, R. Motwani, and L. O'Callaghan. Clustering data streams. In IEEE Symposium on Foundations of Computer Science, pages 359-366, 2000.

22. S. Guha, R. Rastogi, and K. Shim. Cure: an efficient clustering algorithm for large datasets. In Proceedings of ACM SIGMOD International Conference on Management of Data, pages 73-84, 1998.

23. B. Haasdonk and C. Bahlmann. Learning with distance substitution kernels. In Pattern Rcognition - Proc. of the 26th DAGM Symposium, 2004.

24. B. Hammer and A. Hasenfuss. Relational neural gas. In J. Hertzberg, M. Beetz, and R. Englert, editors, KI 200\%: Advances in Artificial Intelligence, 30th Annual German Conference on AI, KI 200\%, volume 4667 of Lecture Notes in Artificial Intelligence, pages 190-204, Berlin, 2007. Springer.

25. B. Hammer and B. Jain. Neural methods for non-standard data. In M. Verleysen, editor, European Symposium on Artificial Neural Networks'2004, pages 281-292. D-side publications, 2004.

26. B. Hammer, A. Micheli, A. Sperduti, and M. Strickert. Recursive self-organizing network models. Neural Networks, 17(8-9):1061-1086, 2004.

27. B. Hammer and T. Villmann. Classification using non standard metrics. In M. Verleysen, editor, ESANN'05, pages 303-316. d-side publishing, 2005. 
28. P. Hansen and M. Mladenovic. Todo. Location Science, 5:207, 1997.

29. A. Hasenfuss and B. Hammer. Single pass clustering and classification of large dissimilarity datasets. In AIPR. 2008.

30. R. J. Hathaway and J. C. Bezdek. Nerf c-means: Non-euclidean relational fuzzy clustering. Pattern Recognition, 27(3):429-437, 1994.

31. R. J. Hathaway, J. W. Davenport, and J. C. Bezdek. Relational duals of the c-means algorithms. Pattern Recognition, 22:205-212, 1989.

32. T. Heskes. Self-organizing maps, vector quantization, and mixture modeling. IEEE Transactions on Neural Networks, 12:1299-1305, 2001.

33. T. Hofmann and J. M. Buhmann. Pairwise data clustering by deterministic annealing. IEEE Transactions on Pattern Analysis and Machine Intelligence, 19(1):1-14, 1997.

34. R. Jin, A. Goswami, and G. Agrawal. Fast and exact out-of-core and distributed k-means clustering. Knowledge and Information System, 1:17-40, 2006.

35. A. Juan and E. Vidal. On the use of normalized edit distances and an efficient $\mathrm{k}-\mathrm{nn}$ search technique (k-aesa) for fast and accurate string classification. In ICPR 2000, volume 2, pages 680-683, 2000.

36. S. Kaski, J. Nikkilä, M. Oja, J. Venna, P. Törönen, and E. Castren. Trustworthiness and metrics in visualizing similarity of gene expression. BMC Bioinformatics, 4, 2003.

37. S. Kaski, J. Nikkilä, E. Savia, and C. Roos. Discriminative clustering of yeast stress response. In U. Seiffert, L. Jain, and P. Schweizer, editors, Bioinformatics using Computational Intelligence Paradigms, pages 75-92. Springer, 2005.

38. L. Kaufman and P. J. Rousseeuw. Clustering by means of medoids. In Y. Dodge, editor, Statistical Data Analysis Based on the L1-Norm and Related Methods, pages 405-416. North-Holland, 1987.

39. T. Kohonen. Self-Organizing Maps. Springer, 1995.

40. T. Kohonen. Self-organizing maps of symbol strings. Technical report A42, Laboratory of computer and information science, Helsinki University of technology, Finland, 1996.

41. T. Kohonen and P. Somervuo. How to make large self-organizing maps for nonvectorial data. Neural Networks, 15:945-952, 2002.

42. A. H. Land and A. G. Doig. An automatic method for solving discrete programming problems. Econometrica, 28:497-520, 1960

43. V. I. Levenshtein. Binary codes capable of correcting deletions, insertions and reversals. Sov. Phys. Dokl., 6:707-710, 1966.

44. Y. Lu, S. Lu, F. Fotouhi, Y. Deng, and S. Brown. Incremental genetic k-means algorithm and its application in gene expression data analysis. BMC Bioinformatics, $5: 172,2004$.

45. C. Lundsteen, J. Phillip, and E. Granum. Quantitative analysis of 6985 digitized trypsin G-banded human metaphase chromosomes. Clinical Genetics, 18:355-370, 1980.

46. T. Martinetz, S. Berkovich, and K. Schulten. 'neural-gas' network for vector quantization and its application to time-series prediction. IEEE Transactions on Neural Networks, 4:558-569, 1993.

47. T. Martinetz and K. Schulten. Topology representing networks. Neural Networks, 7(507-522), 1994.

48. H. Mevissen and M. Vingron. Quantifying the local reliability of a sequence alignment. Protein Engineering, 9:127-132, 1996.

49. M. Neuhaus and H. Bunke. Edit distance-based kernel functions for structural pattern classification. Pattern Recognition, 39(10):1852-1863, 2006. 
50. P.S.Bradley, U.Fayyad, and C.Reina. Scaling clustering algorithms to large data sets. In Proceedings of the Fourth International Conference on Knowledge Discovery and Data Mining, pages 9-15. AAAI Press, 1998.

51. A. Qin and P. Suganthan. Kernel neural gas algorithms with application to cluster analysis. In ICPR 2004, volume 4, pages 617-620. 2004.

52. F. Rossi. Model collisions in the dissimilarity SOM. In Proceedings of XVth European Symposium on Artificial Neural Networks (ESANN 2007), pages 25-30, Bruges (Belgium), April 2007.

53. R. Shamir and R. Sharan. Approaches to clustering gene expression data. In T. Jiang, T. Smith, Y. Xu, and M. Zhang, editors, Current Topics in Computational Biology. MIT press, 2001.

54. T. Villmann, U. Seiffert, F.-M. Schleif, C. Brüß T. Geweniger, and B. Hammer. Fuzzy labeled self-organizing map with label-adjusted prototypes. In F. Schwenker, editor, Proceedings of Conference Articial Neural Networks in Pattern Recognition (ANNPR) 2006, pages 46-56. Springer, 2006.

55. W. Wang, J. Yang, and R. Muntz. Sting: a statistical information grid approach to spatial data mining. In Proceedings of the 23rd VLDB Conference, pages 186-195, 1997.

56. W. Wolberg, W. Street, D. Heisey, and O. Mangasarian. Computer-derived nuclear features distinguish malignant from benign breast cytology. Human Pathology, 26:792-796, 1995.

57. Q. Yang and X. Wu. 10 challenging problems in data mining research. International Journal of Information Technology \& Decision Making, 5(4):597-604, 2006.

58. T. Zhang, R. Ramakrishnan, and M. Livny. Birch: an efficient data clustering method for very large databases. In Proceedings of the 15th ACM SIGACTSIGMOD-SIGART Symposium on Principles of Databas Systems, pages 103-114, 1996. 

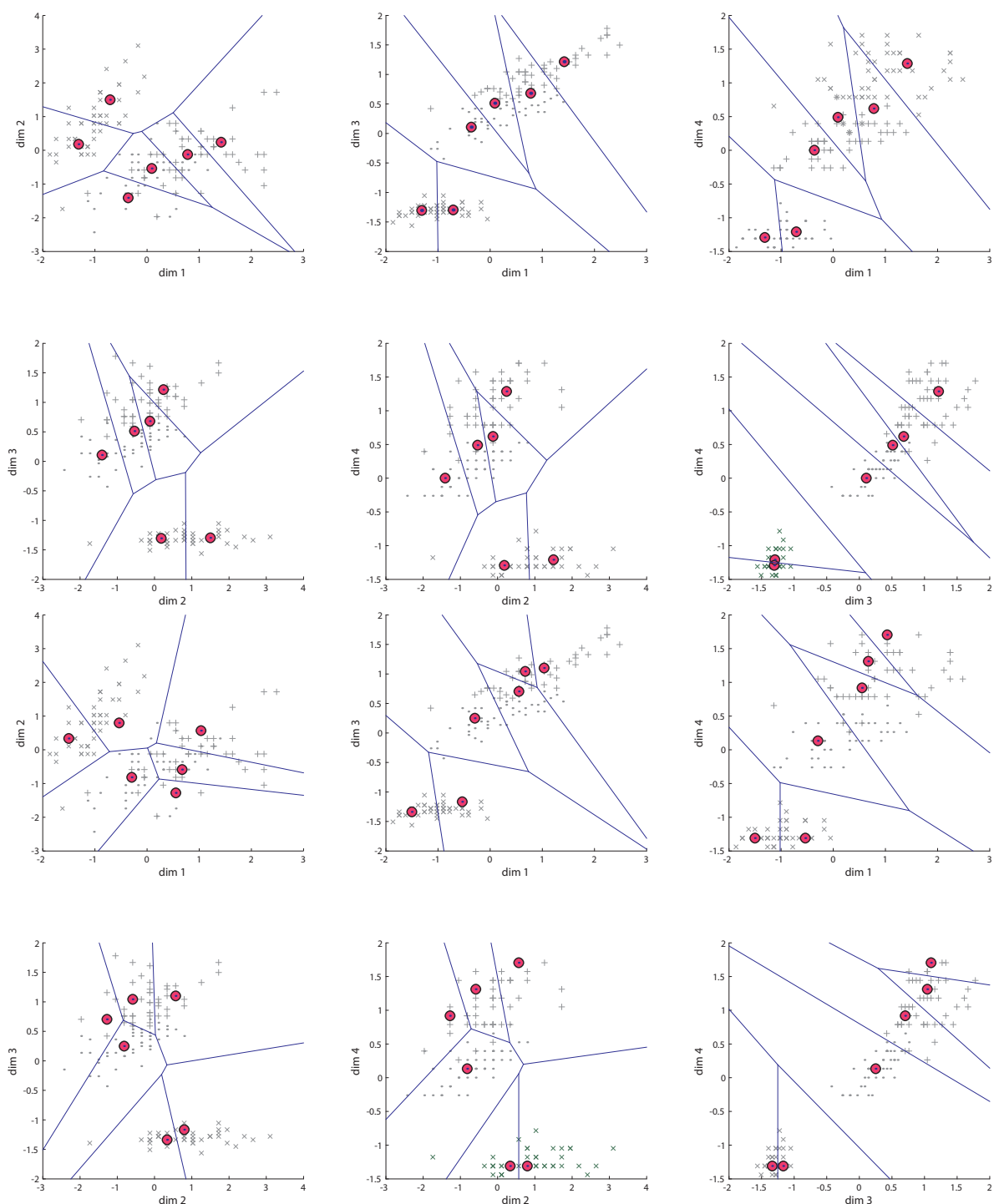

Fig. 1. Results of batch NG (top) and median NG (bottom) on the iris data set projected to two of the four data dimensions. 


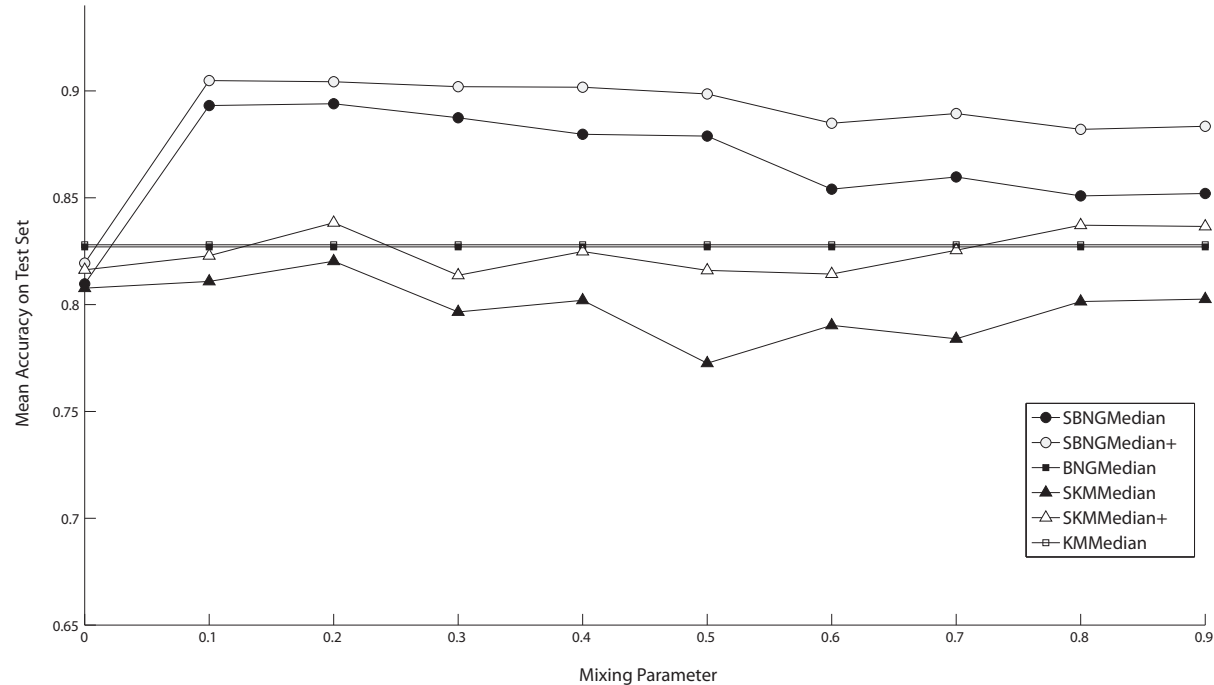

Fig. 2. Results of the methods for the protein database using alignment distance and varying mixing parameter $\beta$. The version indicated with + refers to (optimum) posterior labeling of prototypes. 